\title{
Magnetic relaxation in a classical spin chain
}

\author{
D. Hinzke* and U. Nowak ${ }^{\dagger}$ \\ Theoretische Tieftemperaturphysik, Gerhard-Mercator-Universität-Duisburg, D-47048 Duisburg, Germany
}

\begin{abstract}
With decreasing particle size, different mechanisms dominate the thermally activated magnetization reversal in ferromagnetic particles. We investigate some of these mechanisms for the case of a classical Heisenberg spin chain driven by an external magnetic field. For sufficiently small system size the magnetic moments rotate coherently. With increasing size a crossover to a reversal due to soliton-antisoliton nucleation sets in. For even larger systems many of these soliton-antisoliton pairs nucleate at the same time. These effects give rise to a complex size dependence of the energy barriers and characteristic time scales of the relaxation. We study these quantities using Monte Carlo simulations as well as a direct integration of the Landau-Lifshitz-Gilbert equation of motion with Langevin dynamics and we compare our results with asymptotic solutions for the escape rate following from the Fokker-Planck equation. Also, we investigate the crossover from coherent rotation to soliton-antisoliton nucleation and multidroplet nucleation, especially its dependence on the system size, the external field, and the anisotropy of the system.
\end{abstract}

\section{INTRODUCTION}

Many interesting physical effects occur in connection with the decreasing size of the systems which are under investigation. Magnetic materials are now controllable in the nanometer regime and there is a broad interest in the understanding of the magnetism of small magnetic structures and particles due to the broad variety of industrial applications. ${ }^{1}$ Magnetic particles which are small enough to be singledomain are proposed to have good qualities for magnetic recording and arrays of isolated, nanometer-sized particles are thought to enhance the density of magnetic storage. But on the other hand there is an ultimate limit for the density of magnetic storage which is given by that size of the particles below which superparamagnetism sets in. ${ }^{2}$ Therefore the role of thermal activation for the stability of the magnetization in nanometer-sized structures and particles is studied at present experimentally as well as theoretically.

Wernsdorfer and co-workers measured the switching times in isolated nanometer-sized particles, ${ }^{3,4}$ and wires., 5 For small enough particles ${ }^{3}$ they found agreement with the theory of $\mathrm{Néel}^{7}$ and Brown ${ }^{8}$ who described the magnetization switching in Stoner-Wohlfarth particles by thermal activation over a single energy barrier following from coherent rotation of the magnetic moments of the particle. For larger particles $^{4}$ and also for wires ${ }^{5,6}$ nucleation processes and domain-wall motion were found to become relevant.

Asymptotic formulas for the escape rates following from corresponding Fokker-Planck equations have been derived for ensembles of isolated Stoner-Wohlfarth particles ${ }^{7-12}$ as well as for a one-dimensional model. ${ }^{13,14,10,15}$

Most of the numerical studies of the magnetization switching have been based on Monte Carlo methods. Here, mainly nucleation phenomena in Ising models have been studied ${ }^{16-21}$ but also vector spin models have been used $^{22,23,20,21}$ to investigate the magnetization reversal in systems with many, continuous degrees of freedom qualitatively. However, Monte Carlo methods-even though well established in the context of equilibrium thermodynamics - do not allow for a quantitative interpretation of the results in terms of a realistic dynamics. Only recently, a Monte Carlo method with a quantified time step was introduced. ${ }^{24}$ Here, the interpretation of a Monte Carlo step as a realistic time interval was achieved by a comparison of one step of the Monte Carlo process with a time interval of a Langevin equation, i.e., a stochastic Landau-LifshitzGilbert equation.

Numerical methods for the direct integration of a Langevin equation $^{25,26}$ are more time consuming than a Monte Carlo method but nevertheless highly desirable since here, naturally, a realistic time is introduced by the equation of motion. The validity of different integration schemes is still under discussion (see Ref. 26 for a discussion of the validity of Itô and Stratonovich integration schemes) and hence here, as well as for the Monte Carlo methods, the investigation of analytically solvable models as test tools for the evaluation of the numerical techniques are desirable.

In this paper we will consider a chain of classical magnetic moments - a system which was interpreted as a simplified model for ferromagnetic nanowires or extremely elongated nanoparticles. ${ }^{13}$ Even though arguments against this interpretation have been brought forward ${ }^{27}$ this model is very useful for two reasons: (i) since it was treated analytically asymptotic results for the energy barriers as well as for the escape rates are available. Hence the model can be used as a test tool for numerical techniques. (ii) Depending on the system size, given anisotropies and the strength of the magnetic field different reversal mechanisms may appear and can be investigated. For small system sizes the magnetic moments are expected to rotate coherently, ${ }^{7}$ while for sufficiently large system sizes the so-called soliton-antisoliton nucleation is proposed. ${ }^{13}$

Therefore a systematic numerical investigation of the relevant energy barriers, time scales, and of the crossover from coherent rotation to nucleation is possible.

The outline of the paper is as follows. In Sec. II first we introduce the model and we compare the two different numerical techniques mentioned above, namely the Monte 


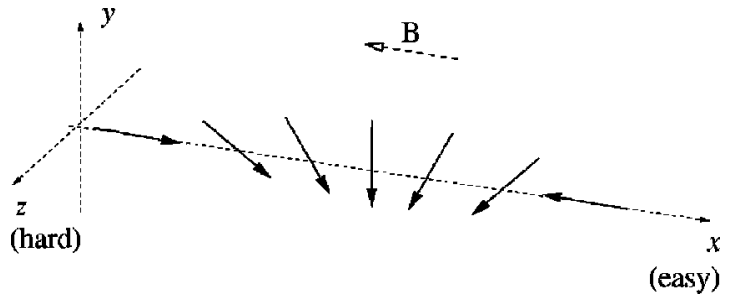

FIG. 1. Sketch of the spin chain. A domain wall is shown where the magnetic moments rotate in the $x-y$ plane. The field is antiparallel to the (easy) $x$ axis.

Carlo method and the numerical solution of the Langevin equation, both of which we will use throughout the paper. In Sec. III we compare our numerical results with the theoretical considerations of Braun, concerning the energy barriers as well as the characteristic times for magnetization switching by coherent rotation as well as by soliton-antisoliton nucleation. For higher temperatures or driving fields, we also find a crossover to multidroplet nucleation, similar to what is known from Ising models. In Sec. IV we summarize our results and relate them to experimental work.

\section{MODEL AND METHODS}

\section{A. Model}

Our intention is to compare our numerical results with Braun's analytical work which bases on a continuum model. For our numerical investigations we use a discretized version of this model namely a one-dimensional classical Heisenberg model. We consider a chain of magnetic moments of length $L$ (number of spins) with periodical boundary conditions defined by the Hamiltonian

$$
E=-J \sum_{\langle i j\rangle} \mathbf{S}_{i} \cdot \mathbf{S}_{j}-d_{x} \sum_{i}\left(S_{i}^{x}\right)^{2}+d_{z} \sum_{i}\left(S_{i}^{z}\right)^{2}-\mu_{s} \mathbf{B} \cdot \sum_{i} \mathbf{S}_{i},
$$

where the $\mathbf{S}_{i}=\boldsymbol{\mu}_{i} / \mu_{s}$ are three-dimensional magnetic moments of unit length. The first sum which represents the exchange of the magnetic moments is over nearest-neighbor interactions with the exchange coupling constant $J$. The second and third sum represent uniaxial anisotropies of the system where the $x$ axis is the easy axis and the $z$ axis the hard axis of the system (anisotropy constants $d_{x}=0.1 J, d_{z}=J$ ). These anisotropy terms may contain contributions from shape anisotropy as well as crystalline anisotropies. ${ }^{15}$ Even though an exact treatment of dipolar interactions would be desirable we let this problem for future work so that our results presented here are comparable to the analytical work of Braun. The last sum is the coupling of the moments to an external magnetic field, where $\mathbf{B}$ is the induction.

Figure 1 shows a sketch of the model. All our simulations using the two different methods above start with a configuration where all magnetic moments point into the $x$ direction, antiparallel to the external magnetic field $\mathbf{B}=-B_{x} \hat{\mathbf{x}}$.

The characteristic time $\tau$ when the $x$ component of the magnetization changes its sign averaged over many simulation runs (100-1000, depending on system size and simulation method) is the most important quantity which we determine. In the case where the temperatures is low compared to the energy barrier the system is in the metastable, initial state for a very long time while the time needed for the magnetization reversal itself is comparatively short. In this case $\tau$ is comparable to the reciprocal of the escape rate, the escape time, which has been calculated for the model considered here asymptotically from the Fokker-Planck equation in certain limits (for details see Ref. 14).

In the classical nucleation theory the so-called lifetime or nucleation time is the time required by the system to build a supercritical droplet which from then on will grow systematically and reverse the system. The metastable lifetime was measured numerically in Ising models by use of similar methods ${ }^{16-21}$. For low enough temperature $T$ all the different times mentioned above are expected to coincide.

The situation is different for higher temperatures where the characteristic time is shorter and the time for the systematic growth of the supercritical droplet is comparable or larger than the nucleation time. Then the characteristic time, i.e., the time the system needs to change its magnetization can be calculated with the aid of the classical nucleation theory (for details see Sec. III C).

\section{B. Langevin dynamics}

The equation describing the dynamics of a system of magnetic moments is the Landau-Lifshitz-Gilbert (LLG) equation of motion with Langevin dynamics. This equation has the form

$$
\begin{aligned}
\frac{\left(1+\alpha^{2}\right) \mu_{s}}{\gamma} \frac{\partial \mathbf{S}_{i}}{\partial t}= & -\mathbf{S}_{i} \times\left(\zeta_{i}(t)-\frac{\partial E}{\partial \mathbf{S}_{i}}\right) \\
& -\alpha \mathbf{S}_{i} \times\left[\mathbf{S}_{i} \times\left(\zeta_{i}(t)-\frac{\partial E}{\partial \mathbf{S}_{i}}\right)\right],
\end{aligned}
$$

with the gyromagnetic ratio $\gamma=1.76 \times 10^{11}(\mathrm{Ts})^{-1}$ and the dimensionless damping constant $\alpha$. The first part of Eq. (2) describes the spin precession while the second part includes the relaxation of the moments. In both parts of this equation the thermal noise $\zeta_{i}(t)$ is included representing thermal fluctuations, with $\left\langle\boldsymbol{\zeta}_{i}(t)\right\rangle=0$ and $\left\langle\boldsymbol{\zeta}_{i}^{k}(t) \boldsymbol{\zeta}_{j}^{l}\left(t^{\prime}\right)\right\rangle=2 \delta_{i j} \delta_{k l} \delta(t$ $\left.-t^{\prime}\right) \alpha k_{\mathrm{B}} T \mu_{s} / \gamma$, where $i, j$ denote the lattice sites and $k, l$ the cartesian components. Equation (2) is solved numerically using the Heun method which corresponds to the Stratonovich discretization scheme. ${ }^{26}$ Note that since the Langevin dynamics simulations are much more time consuming than the Monte Carlo simulation we use this method here mainly for comparison and we will present only data for the relaxation on shorter time scales.

\section{Monte Carlo simulations}

It is inconceivable that the Langevin dynamics simulation can be used over the whole time scale of physical interest so that we simulate the system also using Monte Carlo methods ${ }^{28}$ with a heat-bath algorithm. Furthermore, we use a single spin flip algorithm.

Monte Carlo approaches in general have no physical time associated with each step, so that an unquantified dynamic behavior is represented. However, we use a time quantified Monte Carlo method which was proposed in Ref. 24 where the interpretation of a Monte Carlo step as a realistic time 


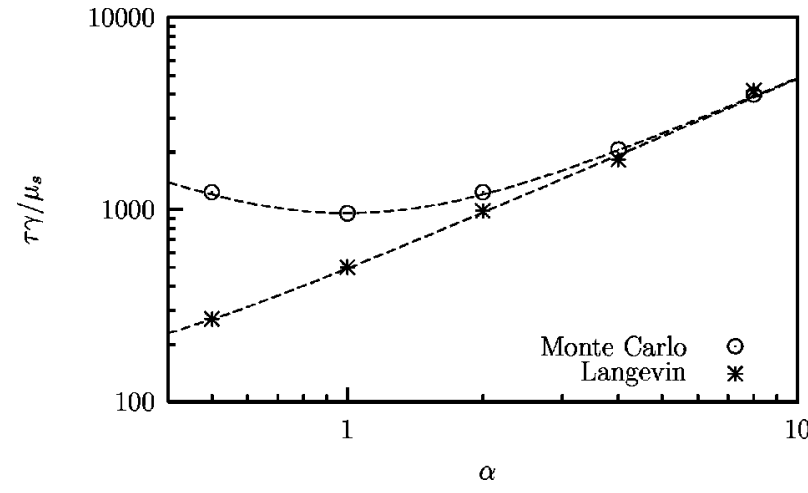

FIG. 2. Reduced characteristic time $\tau \gamma / \mu_{s}$ vs damping constant $\alpha$. The data are from Monte Carlo and Langevin dynamics simulations for $k_{\mathrm{B}} T=0.025 J$ and $L=80$. Dashed lines are guides to the eye.

interval was achieved by a comparison of one step of the Monte Carlo process with a time interval of the LLG equation in the high damping limit. We will use this algorithm in the following. The trial step of this algorithm is a random movement of the magnetic moment within a cone with a given size $r$ with

$$
r^{2}=\frac{20 k_{B} T \alpha \gamma}{\left(1+\alpha^{2}\right) \mu_{s}} \Delta t
$$

In order to achieve this efficiently we construct a random vector with constant probability distribution within a sphere of radius $r$. This random vector is added to the initial moment and subsequently the resulting vector is normalized. ${ }^{24}$

Using this algorithm one Monte Carlo step represents a given time interval $\Delta t$ of the LLG equation in the high damping limit as long as $\Delta t$ is chosen appropriately (for details see Ref. 24).

To test the algorithm, in Fig. 2 the $\alpha$ dependence of the characteristic time of the Monte Carlo data is compared with the data of the Langevin dynamics simulation. Each data point is an average over 1000 independent runs. For low values of the damping constant the data do not coincide while in the high damping limit Monte Carlo and Langevin dynamics data converge. Hence throughout this work we will use $\alpha=4$, which is large enough so that our Monte Carlo simulation and the numerical solution of Eq. (2) yield identical time scales. Even though this is an unphysically large value for $\alpha$ we can compare our results with the analytically obtained high damping asymptotes.

\section{RESULTS}

We investigate the influence of the system size on the occurring reversal mechanisms. There are two extreme cases of reversal mechanisms which might occur in our model namely coherent (or uniform) rotation and nucleation. For small system sizes all magnetic moments of the particle rotate uniformly in order to minimize the exchange energy. For larger system sizes it is favorable for the system to divide into parts of opposite directions of magnetization parallel to the easy axis which minimizes the anisotropy energy. This is a magnetization reversal driven by nucleation and subsequent domain wall propagation. The crossover between these mechanisms is discussed later in this section. First, we study the different reversal mechanism and compare our numerical data with theoretical formulas.

\section{A. Coherent rotation}

In the case of small chain length the magnetic moments rotate coherently while overcoming the energy barrier which is due to the anisotropy of the system. The first theoretical description of the coherent rotation of elongated singledomain particles was developed by Stoner and Wohlfarth. ${ }^{29}$ The belonging energy barrier is

$$
\Delta E_{\mathrm{cr}}=L d_{x}(1-h)^{2}
$$

with $h=\mu_{s} B_{x} /\left(2 d_{x}\right)$. Néel expanded this model for the case of thermal activation ${ }^{7}$ and Brown $^{8}$ calculated the escape time

$$
\tau=\tau_{\mathrm{cr}}^{*} \exp \frac{\Delta E_{\mathrm{cr}}}{k_{\mathrm{B}} T}
$$

following a thermal activation law. The prefactor $\tau_{\mathrm{cr}}^{*}$ is not a simple constant attempt frequency as claimed frequently by several authors but a complicated function which in general may depend on system size, temperature, field, and anisotropies. For our model it is

$$
\frac{\tau_{\mathrm{cr}}^{*} \gamma}{\mu_{s}}=\frac{\pi\left(1+\alpha^{2}\right) \sqrt{\left[d_{z}(1+h)\right] /\left[d_{x}(1-h)+d_{z}\right]}}{\alpha\left[d_{x}\left(1-h^{2}\right)-d_{z}\right]+\sqrt{\left\{\alpha\left[d_{x}\left(1-h^{2}\right)+d_{z}\right]\right\}^{2}+4 d_{x} d_{z}\left(1-h^{2}\right)}}
$$

as was calculated from the Fokker-Planck equation. ${ }^{10}$

The main assumption underlying all results above is that the system can be described by a single degree of freedom, namely the orientation of the magnetic moment of the particle which must have a constant absolute value. Then, the equations above should hold for low enough temperatures $k_{\mathrm{B}} T \ll \Delta E_{\text {cr }}$ and for $\mu_{\mathrm{s}} B_{x}<2 d_{x}$. For larger fields the energy barrier is zero, so that the reversal is spontaneous without thermal activation (nonthermal magnetization reversal).
In the following, our numerical results for the characteristic times $\tau$ for coherent rotation are compared with the equations above. Figure 3 shows the temperature dependence of $\tau$ for a given value of the external magnetic field $h$ $=0.75$ and three different system sizes. For temperatures $k_{\mathrm{B}} T<\Delta E_{\mathrm{cr}}$ our data confirm the asymptotic solutions above for smaller system sizes.

For the largest system shown here $(L=16)$ the numerical data are systematically lower than the theoretical prediction. 


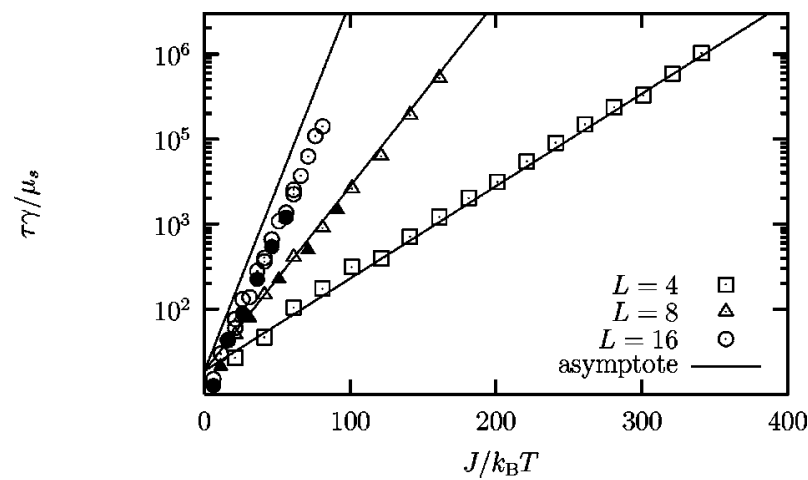

FIG. 3. Reduced characteristic time $\tau \gamma / \mu_{s}$ vs inverse temperature. The data are from Monte Carlo (open symbols) and Langevin dynamics (filled symbols) simulations for different system sizes. The solid lines are the asymptotic formulas for the escape times [see Eqs. (5) and (6)].

Obviously, the prefactor $\tau_{\text {cr }}^{*}$ depends on the system size in contradiction to Eq. (6) while the energy barrier [Eq. (4)] is obviously still correct (the slope of the data). This size dependence of $\tau_{\text {cr }}^{*}$ can be explained with the temperature dependence of the absolute value of the magnetic moment of the extended system. In an extended system, the energy barrier for vanishing field, $d_{x} L$, is reduced to $d_{x}\left\langle\Sigma_{i}\left(S_{i}^{x}\right)^{2}\right\rangle$ which expanded to first order of the temperature can lead to corrections of the form $d_{x}\left\langle\sum_{i}\left(S_{i}^{x}\right)^{2}\right\rangle \approx d_{x} L\left(1-a k_{\mathrm{B}} T\right)$. Including this in Eq. (5) remarkably leads to an effective reduction of the prefactor $\tau_{\mathrm{cr}}^{*}$ by a factor $\exp \left(-a d_{x} L\right)$ and not to a reduction of the effective energy barrier $\Delta E_{\mathrm{cr}}$. To conclude, in extended systems with many degrees of freedom the reduction of the magnetization leads effectively to a size dependent reduction of the prefactor of the thermal activation law for low temperatures.

\section{B. Soliton-antisoliton nucleation}

With increasing length of the chain a different reversal mechanism becomes energetically favorable, namely the socalled soliton-antisoliton nucleation proposed by Braun. ${ }^{14}$ Here, during the reversal the system splits into two parts with opposite directions of magnetization parallel to the easy axis. These two parts are separated by two domain walls with opposite directions of rotation in the easy $x-y$ plane (a soliton-antisoliton pair) which pass the system during the reversal.

Note that we consider periodic boundary conditions, otherwise also the nucleation of one single (anti)soliton at one end of the chain would be possible. The energy barrier $\Delta E_{\text {nu }}$ which has to be overcome during this nucleation process is ${ }^{14}$

$$
\Delta E_{\mathrm{nu}}=4 \sqrt{2 J d_{x}}(\tanh R-h R),
$$

with $R=\operatorname{arcosh}(\sqrt{1 / h})$. In the limit of small magnetic fields, $h \rightarrow 0$, this energy barrier has the form $\Delta E_{\mathrm{nu}}=4 \sqrt{2 J d_{x}}$ which represents the well-known energy of two domain walls. The corresponding characteristic time follows once again a thermal activation law [see Eq. 5]. Since our Monte Carlo simulations are for rather large damping, $\alpha=4$, for comparison of our numerical data we use the prefactor obtained in the overdamped limit [Eq. (5.4) in Ref. 14] which in our units is

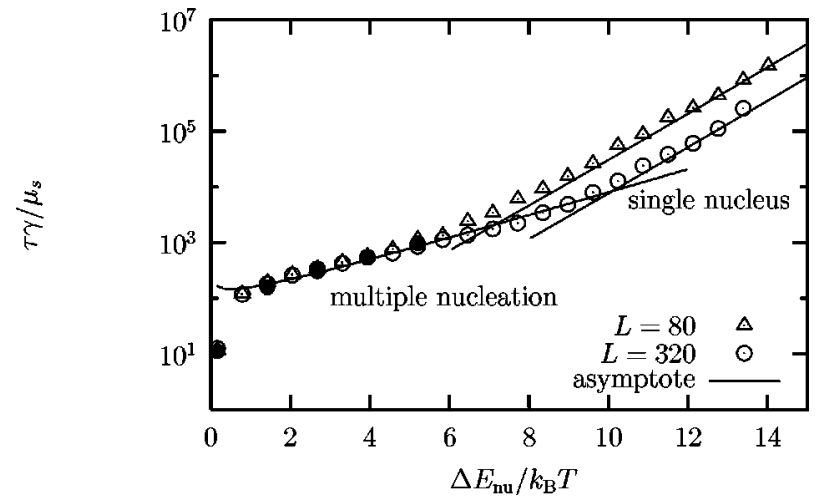

FIG. 4. Reduced characteristic time $\tau \gamma / \mu_{s}$ vs inverse temperature $\Delta E_{\mathrm{nu}} / k_{\mathrm{B}} T$ for two different system sizes. The data are from Monte Carlo (open symbols) and Langevin dynamics (filled symbols) simulations. The solid lines are the asymptotic formulas for the escape time for a single soliton-antisoliton nucleation process [see Eqs. (5), (7), and (8)] and multiple nucleation [see Eq. (11)].

$$
\frac{\tau_{\mathrm{nu}}^{*} \gamma}{\mu_{s}}=\frac{\pi^{3 / 2}\left(1+\alpha^{2}\right)\left(k_{\mathrm{B}} T\right)^{1 / 2}(2 J)^{1 / 4}}{16 \alpha L d_{x}^{7 / 4}\left|E_{0}(R)\right| \tanh R^{3 / 2} \sinh R} .
$$

The eigenvalue $E_{0}(R)$ has been calculated numerically in Ref. 14. In the limit $h \rightarrow 1$ it is $\left|E_{0}(R)\right| \approx 3 R^{2}$. The $1 / L$ dependence of the prefactor reflects the size dependence of the probability of nucleation. The larger the system the more probable is the nucleation process. Furthermore, the prefactor has a remarkable $\sqrt{T}$ dependence leading to a slight curvature in the semilogarithmic plot of the thermal activation law [see Eq. (5)].

Figure 4 shows the temperature dependence of the reduced characteristic time for $h=0.75$ and two different system sizes. The formulas above are confirmed only for rather low temperatures $\left(\Delta E_{\mathrm{nu}} / k_{\mathrm{B}} T>8\right.$ for the smaller system and $\Delta E_{\mathrm{nu}} / k_{\mathrm{B}} T>10$ for the larger system). In the range of intermediate temperatures (but still $k_{\mathrm{B}} T<\Delta E_{\mathrm{nu}}$ ) the numerical data of both, Langevin dynamics and Monte Carlo simulations deviate from the formulas above. Interestingly, in this region the characteristic times do not depend on system size in contrast to Eq. (8) where a $1 / L$ dependence occurs due to the size dependence of the nucleation probability.

In order to understand this effect occurring in the intermediate temperature range, Fig. 5 shows the $x$ component of the magnetic moments of the chain at the characteristic time $\tau$. The upper diagram shows the pure soliton-antisoliton nucleation occurring at low temperatures. Due to thermal fluctuations a nucleus is originated and two domain walls pass the system. The lower diagram shows that alternatively also several nuclei may grow simultaneously. Obviously, depending on temperature (and also other quantities, like system size and field) with a certain probability many nuclei may arise at the same time. This is a multiple nucleation which was investigated mainly in the context of Ising models ${ }^{30}$ where it is called multidroplet nucleation.

\section{Multidroplet nucleation}

The characteristic time $\tau_{\mathrm{mn}}$ for the multidroplet nucleation can be calculated with the aid of the classical nucleation theory ${ }^{31,30}$. In the case of multiple nucleation many nuclei 

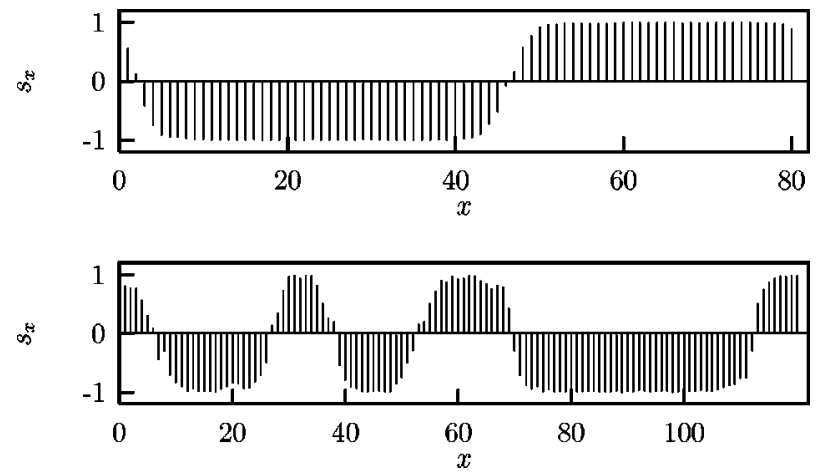

FIG. 5. $x$ component of the magnetic moments of the chain at the time $\tau$ after single soliton-antisoliton nucleation (upper diagram, $\left.L=80, k_{\mathrm{B}} T=0.0019 J, h=0.95\right)$ and multiple nucleation $(L$ $\left.=120, k_{\mathrm{B}} T=0.038 \mathrm{~J}, h=0.95\right)$

with a critical size originate within the same time. These supercritical nuclei grow and join each other leading to the magnetization reversal. The characteristic time for this process is determined by the probability for the occurrence of supercritical nuclei $1 / \tau_{\text {nu }}$ and the time that is needed for the subsequent growth of the nuclei. Let us assume that the radius of a supercritical nucleus grows linearly with the time $t$. Then $\tau_{\mathrm{mn}}$ is given by the condition that the change of the magnetization $\Delta M$ equals the system size, ${ }^{19}$

$$
\Delta M\left(\tau_{\mathrm{mn}}\right)=\int_{0}^{\tau_{\mathrm{mn}}(2 v t)^{D}} \frac{\mathrm{d}}{\tau_{\mathrm{nu}}} \mathrm{d} t=L^{D},
$$

where $v$ is the domain-wall velocity and $D$ the dimension of the system. Hence, the time when half of the system is reversed is given by

$$
\tau_{\mathrm{mn}}=\left(\frac{L}{2 v}\right)^{D /(D+1)}\left[(D+1) \tau_{\mathrm{nu}}^{*}\right]^{1 /(D+1)} \exp \frac{\Delta E_{\mathrm{nu}}}{(D+1) k_{\mathrm{B}} T} .
$$

For the one-dimensional system which we consider in this paper the lifetime is given by

$$
\tau_{\mathrm{mn}}=\sqrt{\frac{L \tau_{\mathrm{nu}}^{*}}{v}} \exp \frac{\Delta E_{\mathrm{nu}}}{2 k_{\mathrm{B}} T} .
$$

This means that the (effective) energy barrier for the multidroplet nucleation is reduced by a factor $1 / 2$ and the prefactor $\tau_{\mathrm{mn}}^{*}$ does no longer depend of the system size since $\tau_{\mathrm{nu}}^{*}$ for the soliton-antisoliton nucleation has a $1 / L$ dependence [see Eq. (8)]. For the comparison in Fig. 4 one also needs the value of the domain-wall velocity which for the case without thermal fluctuations ${ }^{32-34}$ is

$$
v=\frac{\gamma B_{x}}{\alpha} \sqrt{\frac{J}{2 d_{x}}} .
$$

Even though this formula neglects thermal fluctuations we will use it in the following for our asymptotic formulas. The corresponding value of the domain velocity for $B_{x}=0.15 \mathrm{~J}$ in our units is $v=0.038$ spins per reduced time unit.

In Fig. 4 the validity of Eq. (11) is confirmed. Also, it can be seen, that the prefactor is not dependent on the system size since the data for both sizes coincide as is explained by

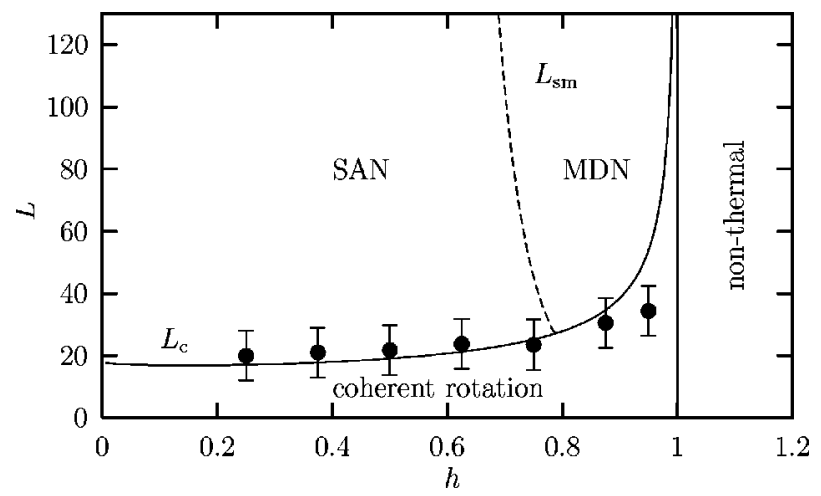

FIG. 6. Diagram showing the regions of different reversal mechanisms. The lines corresponds to Eqs. (14) and (13). The points are data from Monte Carlo simulations confirming Eq. (14).

the considerations above. A similar crossover from single to multidroplet excitations was observed in Ising models, field dependent ${ }^{17-19}$ as well as temperature dependent. ${ }^{21}$ Comparing the characteristic times for single and multiple nucleation we get for the intersection of these two times the crossover condition

$$
L_{\mathrm{sm}}=\sqrt{v \tau_{\mathrm{nu}}^{*} L_{\mathrm{sm}}} \exp \frac{\Delta E_{\mathrm{nu}}}{2 k_{\mathrm{B}} T} .
$$

The corresponding time is $L_{\mathrm{sm}} / v$ - the time that a domain wall needs to pass the system. This results is also comparable to calculations in Ising models. ${ }^{17}$

\section{Crossover}

In this section we are interested in the crossover between coherent rotation and soliton-antisoliton nucleation. Therefore we compare the energy barrier of soliton-antisoliton nucleation [see Eq. 7] with the energy barrier of coherent rotation [see Eq. (4)] in order to obtain a condition for the mechanism with the lowest activation energy. The resulting crossover line $L_{c}$ has the form

$$
L_{c}=\frac{4 \sqrt{2 J}(\tanh R-h R)}{\sqrt{d_{x}}(1-h)^{2}} .
$$

For vanishing magnetic field it is $L_{c}=4 \sqrt{2 J / d_{x}}$. Note that Braun also determined this crossover line from a slightly different condition. ${ }^{35}$ He calculated the system size below which no nonuniform solution for the Euler-Lagrange equations exists and he obtained a limit which is similar to Eq. (14) but a little bit lower.

A diagram showing which reversal mechanisms occur in our model depending on system size and field is presented in Fig. 6. The crossover line $L_{c}$ derived above separates the coherent rotation region from that of soliton-antisoliton nucleation. For $h>1$ the reversal is nonthermal. In the nucleation region, for larger fields a temperature-dependent crossover to multiple nucleation sets in. However, the lower the temperature the more this region vanishes. As an example we show in the figure the line $L_{\mathrm{sm}}$ which separates single from multiple nucleation for $k_{\mathrm{B}} T=0.006 J$.

In order to confirm Eq. (14) numerically we determine the mean correlations of the $y$ components of two spins located 


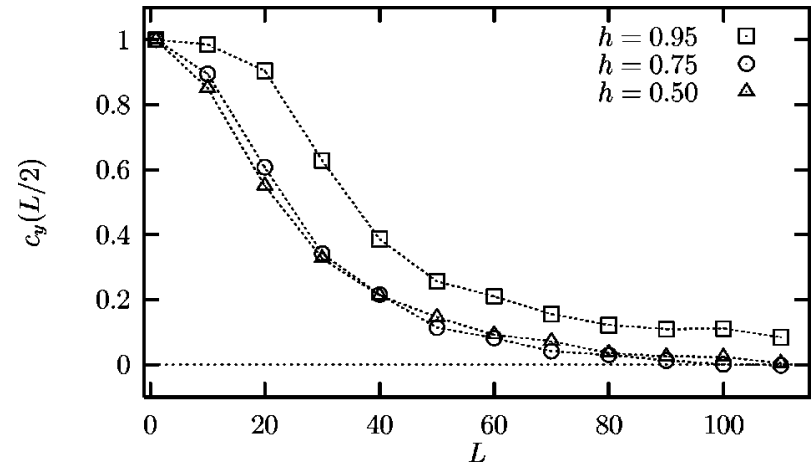

FIG. 7. System size dependence of $c_{y}(L / 2)$ for different magnetic fields. $k_{\mathrm{B}} T=0.1 \cdots 0.004 \mathrm{~J}$ depending on $h$.

in a distance of $L / 2$ (the maximum distance in a system with periodic boundary conditions) at the time $\tau$ during the reversal

$$
c_{y}(L / 2)=\left[\frac{1}{N} \sum_{i=1}^{N} S_{i}^{y}(\tau) S_{i+L / 2}^{y}(\tau)\right]_{\mathrm{av}} .
$$

This quantity characterizes the reversal mechanism. Figure 7 shows the system size dependence of $c_{y}(L / 2)$ for different values of the magnetic field. For coherent rotation the limiting value of $c_{y}(L / 2)$ is 1 since at the time $\tau$ all moments point into the $y$ direction (small systems). On the other hand, for nucleation the system is mainly split into two parts where the moments point into $\pm x$ direction. In this case the correlations in $y$ direction are zero (larger system size). As a criterion for the crossover between nucleation and coherent rotation we use a value of $1 / 2$ for the correlation function and define the corresponding system size as the crossover length. This analysis leads to the numerical data shown in Fig. 6.

The Monte Carlo data confirm the theoretical crossover line except for the largest magnetic field. Here, the numerical data differ from the theoretical crossover line which diverges in the limit $h \rightarrow 1$. In this region a mixed reversal mechanism appears: first the magnetic moments rotate coherently up to a certain rotation angle and then an instability sets in and a (restricted) soliton-antisoliton pair arises. Obviously, the coherent rotation is unstable in this region. A hint for the existence of this mixed mechanism can also be found in Fig. 7: for the field $h=0.95$ the correlation is not zero for large system sizes but remains finite due to the fact that the whole system first rotates by a certain angle before the nucleation sets in.

\section{CONCLUSIONS}

We considered the magnetization switching in a classical Heisenberg spin chain as a model for the study of nucleation processes and as a test tool for numerical techniques since analytical expressions for the relevant energy barriers and time scales exist in several limits. Numerically we used Monte Carlo methods as well as Langevin dynamics simulations and we confirmed that the Monte Carlo algorithm we use yield time quantified data comparable to those of the Langevin dynamics simulation in the limit of high damping.

Varying the system size we observed different reversal mechanisms, calculated the characteristic times for the rever-

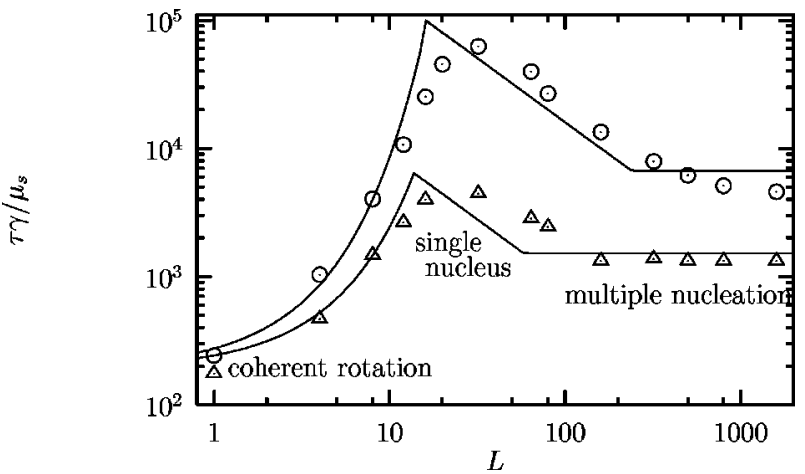

FIG. 8. Reduced characteristic time $\tau \gamma / \mu_{s}$ vs system size $L$ for two different temperatures $\left(k_{\mathrm{B}} T=0.024 J\right.$ and $\left.k_{\mathrm{B}} T=0.016 J\right)$. The solid lines are the appropriate formulas and the data are from Monte Carlo simulations.

sal (i.e., the time scale of the relaxation process) and compared our results with analytical considerations. The comparison of our numerical data with theoretical considerations confirms that both of our numerical techniques can be used to study the relaxation behavior of magnetic systems qualitatively and quantitatively.

Figure 8 summarizes our results. It shows the system size dependence of the reduced characteristic time for two different temperatures. For small system sizes the spins rotate coherently. Here, the energy barrier [Eq. (4)] is proportional to the system size leading to an exponential increase of $\tau$ with system size. Following Eq. (6) the prefactor of the thermal activation law should not be $L$ dependent. However, here we found slight deviations from the asymptotic expressions stemming from the nonconstant magnetization of extended systems. In the regime of soliton-antisoliton nucleation the energy barrier [Eq. (7)] does not depend on the system size but the prefactor $[\mathrm{Eq} .(8)]$ has a $1 / L$ dependency. Interestingly, this leads to a decrease of the characteristic time with increasing system size. Therefore there is a maximum relaxation time close to where the crossover from coherent rotation to nucleation occurs [see Eq. (14)]. This decrease ends where the so-called multidroplet nucleation sets in $[\mathrm{Eq}$. (13)]. From here on with increasing system size the characteristic time remains constant [Eq. (11)].

Note that qualitatively the same behavior can be found in the particle size dependence of the dynamic coercivity. Solving the equation describing the thermal activation [Eq. (5)] in the three regimes explained above for $h(L, \tau=$ const $)$, one finds an increase of the coercivity in the coherent rotation regime, a decrease in the nucleation regime, and at the end a constant value for multiple nucleation. This is qualitatively in agreement with measurements of the size dependence of barium ferrite recording particles. ${ }^{36}$

We should mention that the model treated here was suggested as simplified model for magnetic nanowires. However, it contains the magnetostatic energy only in a local approximation. ${ }^{15}$. Arguments against this treatment have been brought forward ${ }^{27}$ so that the question whether an explicit inclusion of the dipole-dipole interaction would lead to additional reversal modes (e.g., curling)_also depending on wether the diameter of the nanowire is larger or smaller than the exchange length-remains open. Calculations following these lines are therefore highly desirable. 
Comparing the energy barrier for the nucleation process with experimental values [Eq. (7)], one should also mention that in an experimental system nucleation usually will start at the sample ends. This will reduce the energy barrier by a factor of 2 since without periodical boundary conditions one does not need a soliton-antisoliton pair but just one single excitation. The energy barrier for the nucleation process [Eq. (7)] was compared in Ref. 15 with energy barriers measured in isolated $\mathrm{Ni}$-nanowires ${ }^{5,6}$ finding the experimental energy barrier reduced by a factor of 3 . This agreement is rather encouraging taking into account that in realistic systems the energy barrier might be reduced depending on the form of the sample ends.

Regarding the crossover from coherent rotation to nucleation a similar analysis, even though less rigorous, has been performed for three-dimensional systems. ${ }^{20}$ Here, in the limit $h \rightarrow 0$ the crossover diameter is $L_{c}=3 / 2 \sqrt{2 J / d_{x}}$ instead of $2 \sqrt{2 J / d_{x}}$ for the spin chain with open boundary conditions. As discussed in Ref. 20 for CoPt particles the crossover length should be of the order of $30 \mathrm{~nm}$ which is also roughly in agreement with experiments on Co particles. ${ }^{3,4}$

\section{ACKNOWLEDGMENTS}

We thank K. D. Usadel and D. A. Garanin for fruitful discussion and $\mathrm{H}$. B. Braun also for providing numerical results for the eigenvalue $\left|E_{0}(R)\right|$. This work was supported by the Deutsche Forschungsgemeinschaft through the Graduiertenkolleg "Struktur und Dynamik heterogener Systeme" and was done within the framework of the COST action P3 working group 4.
*Electronic address: denise@thp.uni-duisburg.de

†Electronic address: uli@thp.uni-duisburg.de

${ }^{1}$ F.J. Himpsel, J.E. Ortega, G.J. Mankey, and R.F. Willis, Adv. Phys. 47, 511 (1998)

${ }^{2}$ R. W. Chantrell and K. O'Grady, in Applied Magnetism, edited by R. Gerber, C. D. Wright, and G. Asti (Kluwer Academic Publishers, Dordrecht, 1994), p. 113.

${ }^{3}$ W. Wernsdorfer, E.B. Orozco, K. Hasselbach, A. Benoit, B. Barbara, N. Demoncy, A. Loiseau, H. Pascard, and D. Mailly, Phys. Rev. Lett. 78, 1791 (1997).

${ }^{4}$ W. Wernsdorfer, K. Hasselbach, A. Sulpice, A. Benoit, J.-E. Wegrowe, L. Thomas, B. Barbara, and D. Mailly, Phys. Rev. B 53, 3341 (1996).

${ }^{5}$ W. Wernsdorfer, B. Doudin, D. Mailly, K. Hasselbach, A. Benoit, J. Meier, J.P. Ansermet, and B. Barbara, Phys. Rev. Lett. 77, 1873 (1996).

${ }^{6}$ W. Wernsdorfer, K. Hasselbach, A. Benoit, B. Barbara, B. Doudin, J. Meier, J.P. Ansermet, and D. Mailly, Phys. Rev. B 55, 11 552 (1997).

${ }^{7}$ L. Néel, Ann. Geophys. (C.N.R.S.) 5, 99 (1949).

${ }^{8}$ W.F. Brown, Phys. Rev. 130, 1677 (1963).

${ }^{9}$ I. Klik and L. Gunther, J. Stat. Phys. 60, 473 (1990).

${ }^{10}$ H.B. Braun, J. Appl. Phys. 76, 6310 (1994).

${ }^{11}$ W.T. Coffey, D.S.F. Crothers, J.L. Dormann, Y.P. Kalmykov, E.C. Kennedy, and W. Wernsdorfer, Phys. Rev. Lett. 80, 5655 (1998).

${ }^{12}$ D.A. Garanin, E.C. Kennedy, D.S.F. Crothers, and W.T. Coffey, Phys. Rev. E 60, 6499 (1999).

${ }^{13}$ H.B. Braun, Phys. Rev. Lett. 71, 3557 (1993).

${ }^{14}$ H.B. Braun, Phys. Rev. B 50, 16485 (1994).

${ }^{15}$ H.B. Braun, J. Appl. Phys. 85, 6127 (1999).

${ }^{16}$ D. Stauffer, Int. J. Mod. Phys. C 3, 1059 (1992).

${ }^{17}$ P.A. Rikvold, H. Tomita, S. Miyashita, and S.W. Sides, Phys. Rev. E 49, 5080 (1994).
${ }^{18}$ P. A. Rikvold, M. A. Novotny, M. Kolesik, and H. L. Richards, in Dynamical Properties of Unconventional Magnetic Systems, edited by A. T. Skjeltrop and D. Sherrington (Kluwer, Dordrecht, 1998), p. 307.

${ }^{19}$ M. Acharyya and D. Stauffer, Eur. Phys. J. B 5, 571 (1998).

${ }^{20}$ D. Hinzke and U. Nowak, Phys. Rev. B 58, 265 (1998).

${ }^{21}$ U. Nowak and D. Hinzke, J. Appl. Phys. 85, 4337 (1999).

22 J.M. González, R. Ramírez , R. Smirnov-Rueda, and J. González, Phys. Rev. B 52, 16034 (1995).

${ }^{23}$ J.M. Gonzáles, R. Smirnov-Rueda, and J. González, J. Appl. Phys. 81, 5573 (1997).

${ }^{24}$ U. Nowak, R.W. Chantrell, and E.C. Kennedy, Phys. Rev. Lett. 84, 163 (2000).

${ }^{25}$ A. Lyberatos and R.W. Chantrell, J. Appl. Phys. 73, 6501 (1993).

${ }^{26}$ J.L. García-Palacios and F.J. Lázaro, Phys. Rev. B 58, 14937 (1998).

${ }^{27}$ A. Aharoni, J. Appl. Phys. 80, 3133 (1996).

${ }^{28}$ K. Binder and D. W. Heermann, in Monte Carlo Simulation in Statistical Physics, edited by P. Fulde (Springer-Verlag, Berlin, 1997), p. 21.

${ }^{29}$ E.C. Stoner and E.P. Wohlfarth, Philos. Trans. R. Soc. London, Ser. A 240, 599 (1949).

${ }^{30}$ P. A. Rikvold and B. M. Gorman, in Annual Reviews of Computational Physics I, edited by D. Stauffer (World Scientific, Singapore, 1994), p. 149.

${ }^{31}$ R. Becker and W. Döring, Ann. Phys. (Leipzig) 24, 719 (1935).

${ }^{32}$ J. F. Dillon, in Magnetism III, edited by G. T. Rado and H. Suhl (Academic Press, London, 1963), p. 450.

${ }^{33}$ A. P. Malozemoff and J. C. Slonczewski, Magnetic Domain Walls in Bubble Materials (Academic Press, London, 1979).

${ }^{34}$ E. Magyari and H. Thomas, Z. Phys. B: Condens. Matter 59, 167 (1985).

${ }^{35}$ H. B. Braun (World Scientific, Singapore, 1999).

${ }^{36}$ T. Chang, J.-G. Zhu, and J.H. Judy, J. Appl. Phys. 73, 6716 (1993). 